ticular cases with which one can enthusiastically agree, or disagree.

ANGUS J MCKAY Department of Philosophy, University of Glasgow

\section{Medical Futility and the Evaluation of Life-sustaining Interventions}

\author{
Edited by $M$ B Zucker and \\ H D Zucker, Cambridge, Cambridge \\ University Press, 1997, 201 pages, \\ $£ 45 \mathrm{hb}, £ 15.95 \mathrm{pb}$.
}

At the American College of Physicians' conference in 1993, it was reported from New York State that of 329 cardiopulmonary resuscitation (CPR) attempts in 307 patients, $65 \%$ had taken place in patients terminally ill with AIDS, cancer, stroke or sepsis. This was presented as the result of state legislation. Most of us find this shocking: shocking in its indignity to the dying, shocking in the suffering for patients and families in the temporary "successes", shocking in waste of resources, shocking in its implications of how we view dying.

Cardiopulmonary resuscitation is a particularly stark example of a lifesustaining intervention, for without it death is inevitable. It is a paradigm case and in any book on life-sustaining interventions it rightly occupies a major place. Successful CPR varies according to setting or patient group from around $50 \%$ to under $1 \%$. Its success is generally overestimated by both the lay public and by health care professionals, especially nurses. Discussion between patients and their carers is the exception rather than the rule, despite exhortations, guidelines, circulars and legislation; written information fails to bridge this gap in the vast majority of cases. Is there then a point at which CPR should be defined as futile by the doctors and not considered? Do patients have a right to useless or ineffectual interventions? If not, what level of probability defines "useless" or "ineffectual"? If CPR is the starkest example, what about every other intervention of doubtful benefit in societies in which third parties pay? Should you pay for me to have a useless treatment just because I do not know or will not accept its almost certain lack of value? As Murphy remarks in his chapter in this book, if our society cannot deal responsibly with futile interventions we have little hope of dealing with any kind of marginally beneficial care. From one perspective, futility is a special case of a wider debate about rationing.

These themes make up the 16 chapters in this collection. Overall "futility" emerges as a necessary concept, albeit one capable of abuse and needing careful definition. Brody's initial chapter elegantly outlines the arguments on both sides and concludes that notions of professional integrity have a pivotal role. Once we accept that the principles of autonomy and justice cannot produce satisfactory solutions to highly practical problems, the way is cleared for futility judgments to start rather than stop conversations. The remaining chapters embroider Brody's themes, inevitably with some repetition, but never challenging his main thesis. A dissenting chapter would have been welcome: not everyone is happy with "futility" and the debate goes beyond defining and applying it.

Brody's chapter is followed by a personal account by Patricia Brophy of Brophy $v$ New England Sinai Hospital. For a British reader, it is instructive to read this in conjunction with the analysis of the public and press response to the Jaymee Bowen case, in which futility arguments were so confused with economics. ${ }^{1}$ The remaining chapters fall into four groups: those considering futility in different groups or settings (intensive care unit, medical wards, nursing homes, children, the old); those on practical aids to conflict resolution (ethics committees, liaison psychiatrists); those on culture, community projects and policies, and those on legal and economic perspectives. Not all are successful in producing a balanced account: seven lines on Islam compared to nearly four times that number on Native American culture $(0.8 \%$ of the US population) in the chapter on religious attitudes is an example. Sometimes essential factual information could be more clearly separated from the ethical debate. Sometimes the treatment is too superficial: under six pages on alternative medicine and medical futility barely scratches the surface. But the best chapters are very good indeed, contributing perspectives from a wide range of disciplines. Brody's initial chapter is an example of the fine writing that we have come to expect from him; Paris and Poorman contribute an account of conflict between medical judgments and religious beliefs whose 'implications are wider than the title; Murphy's account of the economics of futile interventions reveals a wisdom that is the fruit of a mature reflection on these issues and not merely an analysis of costs. If these were the highlights for this reviewer, there is much more that is rewarding to study - and none of it over-lengthy. A valuable contribution to a subject of immense practical importance and warmly recommended.

\section{References}

1 Entwistle VA, Watt IS, Bradbury R, Pehl LJ. Media coverage of the child B case. British Medical fournal 1996;112:1587-91.

JOHN SAUNDERS Department of Medicine, Nevill Hall Hospital, Abergaveny, Centre for Philosophy and Health Care, University of Wales, Swansea

\section{Ethical Dilemmas in Assisted Reproduction}

Edited by F Shenfield and C Sureau, New York and Carnforth, Lancashire, Parthenon Publishing Group Ltd, 1997,96 pages, $£ 39$ hb US $\$ 65$.

This slim but dense volume offers the reader concise discussions on a number of the complex ethical dilemmas presented by assisted reproductive technology.

It is clear that the question of ethics in this field is a moving stream. The reasons why we need an ethical imperative in donor insemination (DI), in in vitro fertilisation (IVF), and in intra-cytoplasmic sperm injection (ICSI), and surrogacy are given. The welfare of the unborn child is widely understood as a matter of the greatest importance. Yet, the needs of the prospective parents cannot be ignored. These ethical values are not those of the medical profession but are based upon the culture of the population they serve; the wide sense of disquiet over the recent birth of a baby to a 60 -year-old being an example of the public's close attention to these practices.

The constant progression of scientific techniques in medicine demands that we continuously re-examine all ethical questions. For example, sex selection and pre-implantation diagnosis offer fresh dilemmas, the resolution of which will have as much to do with the culture of the population as with the clinicians' own views - and 